\title{
Automated Infrastructure for High-Throughput Acquisition of Serial Section TEM Image Volumes
}

\author{
Robinson, C.G. ${ }^{1}$, Price, J. ${ }^{2}$, Milkie, D. ${ }^{3}$, Torrens, O. ${ }^{3}$, Perlman, E. ${ }^{1}$, Zheng, Z. ${ }^{1}$, Fetter, R.D. ${ }^{1}$, Bock, \\ D.D. ${ }^{1}$ \\ 1. Howard Hughes Medical Institute, Janelia Research Campus, Ashburn, VA, USA. \\ 2. Hudson Price Designs, LLC, Hingham, MA, USA. \\ 3. Coleman Technologies, Inc., Newton Square, PA, USA.
}

Electron microscopy (EM) has sufficient resolution to map neural circuits at the level of individual neurons and the chemical synapses between them (i.e. connectomics). However, millions of high resolution EM images ( 4 × 4 × $40 \mathrm{~nm}$ voxel) are needed to span brain volumes of sufficient size to contain circuits of interest. Therefore, high-throughput imaging at high-resolution remains a primary challenge for connectomics [1]. We present here infrastructure to greatly reduce the acquisition time for this class of EM volume, through parallelized image readout, high-speed sample motion during mosaicking, and automated exchange of TEM sample grids.

We designed a second-generation TEM camera array (TEMCA2) based on a FEI Spirit BioTWIN and comprised of a custom vacuum extension, scintillator (Grant Scientific), optical quality leaded vacuum glass (Computer Optics), and 2x2 array of optically coupled 5.5 megapixel sCMOS cameras (Fig. 1a-b). A LabVIEW (National Instruments)-based acquisition control system provides hardware synchronization between a custom piezo-driven fast sample stage (Fig. 1e) and the camera array, enabling rapid acquisition of high quality image mosaics. Acquired data is written over dedicated $10 \mathrm{~GB} / \mathrm{s}$ fiber optic links to a series of data storage servers.

The custom fast stage holds a single $3 \mathrm{~mm}$ slot grid and is compatible with the standard multiple-axis Compustage on the BioTWIN. In typical operation the fast stage steps $8 \mu \mathrm{m}$ (one camera field of view) and settles in 30-50 ms. Four $35 \mathrm{~ms}$ frames per field of view are acquired. Real-time drift analysis is performed by cross-correlating sequential frames, ensuring that samples are stable before images are saved to disk. To increase signal-to-noise, estimated drift information is used to translate and sum frames when inter-frame drift is $\leq 4$ pixels $(\sim 16 \mathrm{~nm})$. If drift is $>4$ pixels, frames are discarded and additional frames are acquired until the desired number of stable frames per field of view have been obtained. On average, the TEMCA2 system acquires data at a net rate of $\sim 50 \mathrm{MB} / \mathrm{sec}$ (including stage motion, FOV overlap, and computational overhead), $\sim 6 \mathrm{x}$ faster than the original TEMCA system [2], while maintaining equally high image contrast and quality (Fig. 1h). The extremely brief acquisition time per frame necessitates large emission current ( 15-40 uA) from the electron gun, and high electron

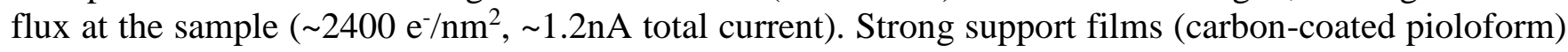
and a minimum of electron-opaque artifacts are required to avoid rupturing the sample support film due to this high electron dose.

We also developed the Autoloader (Fig. 1c), which combines a multi-axis grid-positioning system (GPS; Fig. 1f), with an in-vacuum multi-sample storage magazine consisting of eight cassettes, each of which holds 64 grids (512 grid total capacity). The Autoloader is mounted on an objective lens accessory port, and enables unattended sample exchange and fast-mosaic imaging of custom serially numbered sample grids (Fig. 1d). A vacuum-compatible camera mounted on the GPS gripper (Fig. 1g) enables machine 
vision-guided pick-and-place of grids from the cassette. Once the sample grid is loaded into the TEM, regions of interest may be specified and relocated across acquires, enabling batch acquisition across multiple samples at multiple magnifications. In addition to in-plane translation and z-adjust, the GPS is capable of tilting the sample about its long axis; in the future this tilt capability, in combination with use of the Autoloader's prealigner, may enable 2-axis tomography for sub-nanometer resolution.

This infrastructure has proved reliable and robust. Two TEMs, one with a TEMCA2 array and one with an Autoloader and a single 5.5 MP camera, were used to completely image a serially sectioned brain of a female adult fruit fly Drosophila melanogaster ( 700x350x350 um, 7,060 sections). The dataset comprises $\sim 106 \mathrm{~TB}$ on disk and $\sim 21$ million camera images. The data were acquired over a period of 16 months, mostly using a TEMCA2 system (83\% of imaged sections; 4.3 million fast stage moves). The Autoloader came online during the last quarter of this time period (17\% of imaged sections; 3.5 million GPS moves; 6800 automated pick and place steps). 82\% of Autoloader pick-and-places were successful; of the other $18 \%, \sim 80 \%$ of grids were re-stowed automatically, and $\sim 20 \%$ required manual control of the Autoloader to re-stow (4\% of all grids). Re-stowed grids were imaged manually with a fast stage on a TEMCA2. Zero samples were lost or damaged due to Autoloader or fast stage malfunction. Given this success, these imaging tools remain deployed for ongoing large-scale volume electron microscopy imaging efforts. [3]

\section{References:}

[1] K.L. Briggman and D.D. Bock, Curr Opin Neurobiol, 22 (2012), p. 154.

[2] D.D Bock, et al., Nature, 471 (2011), p. 177.

[3] This work was funded by the Howard Hughes Medical Institute.
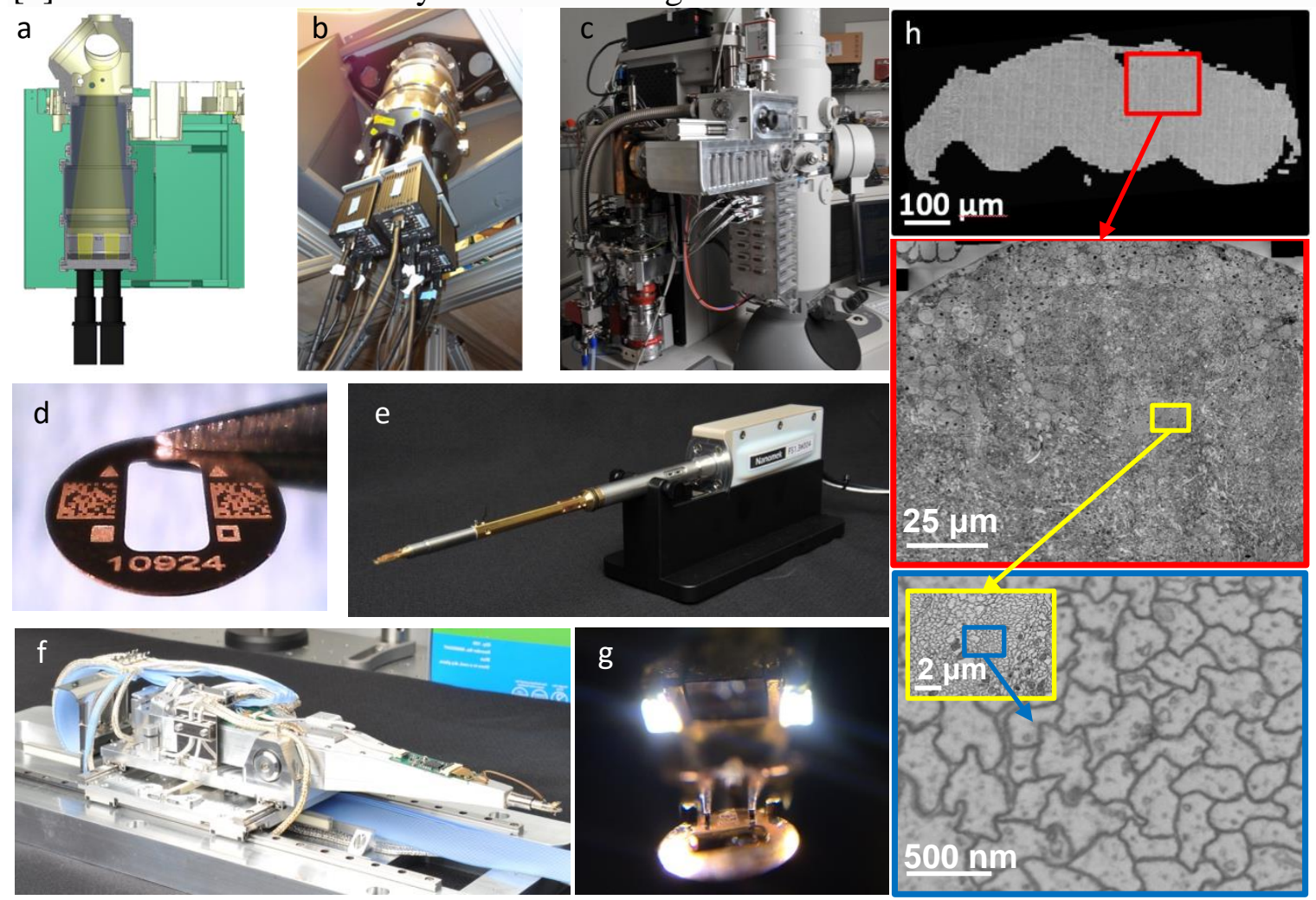

Figure 1. (a \& b) custom vacuum extension and camera array, (c) Autoloader, (d) 2D barcoded grid, (e) fast stage, (f) Autoloader GPS, (h) GPS end effector, (i) mosaic and enlargements. 